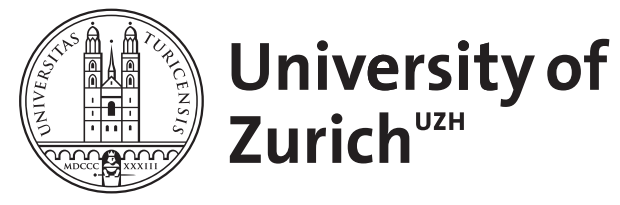

Zurich Open Repository and Archive

University of Zurich

University Library

Strickhofstrasse 39

CH-8057 Zurich

www.zora.uzh.ch

Year: 2012

\title{
Sind bipolare Störungen viel häufiger als bisher angenommen? Pro
}

Angst, J

DOI: https://doi.org/10.1007/s00115-012-3578-6

Posted at the Zurich Open Repository and Archive, University of Zurich

ZORA URL: https://doi.org/10.5167/uzh-156748

Journal Article

Published Version

Originally published at:

Angst, J (2012). Sind bipolare Störungen viel häufiger als bisher angenommen? Pro. Der Nervenarzt, 83(7):903-904.

DOI: https://doi.org/10.1007/s00115-012-3578-6 
Nervenarzt 2012 $\cdot 83: 903-904$

DOI 10.1007/s00115-012-3578-6

Online publiziert: 24. Juni 2012

(c) Springer-Verlag 2012

\author{
J. Angst \\ Forschungsabteilung Psychiatrische, Universitätsklinik Zürich
}

\title{
Sind bipolare Störungen viel häufiger als bisher angenommen? Pro
}

ko bleibt mit steigendem Lebensalter konstant und beträgt ca.1,25\% pro Lebensjahr.

Antidepressiva sind mit einem Umschlag einer Depression in die Hypomanie assoziiert, aber sie verursachen ihn nicht. In doppelblinden Untersuchungen von Depressiven finden sich unter Placebos stark Gebesserte weniger häufig als unter einer wirksamen Substanz. Deshalb ist die Umschlagsrate in die Hypomanie unter Placebos ebenfalls geringer, denn der Umschlag setzt ja eine deutliche Besserung (z. B. Response) voraus und unterliegt daher statistisch dem Bayes-Theorem (bedingte Wahrscheinlichkeit), was bisher in keiner Publikation berücksichtigt wurde. Die Hypomanie unter Antidepressiva ist also keine Nebenwirkung, sondern eine Folge der Wirkung und Besserung der depressiven Phasen.

\section{》) Die versteckten}

unterschwelligen bipolar

Depressiven bilden ein

weltweites Problem

In neuen Analysen großer epidemiologischer Studien wurden hypomanische Syndrome unter der diagnostischen Schwelle in ca. $40 \%$ der Major-Depressionen identifiziert. Diese Ergebnisse stammen aus der EDSP-Studie des MPI München [9] und aus der NCS-R-Studie in den USA [1]. Depressive Menschen mit unterschwelliger Bipolarität unterscheiden sich statistisch hoch signifikant von den rein Depressiven und zeigen eindeutig ein Validierungsprofil von bipolaren Störungen: positive Familienanamnese, früherer Krankheitsbeginn, höhere Periodizität, bessere Remission zwischen den Phasen, höhere Saisonalität und vor allem eine viel höhere Komorbidität mit Angsterkrankungen, Substanzmissbrauch jeder Art sowie höhere Resistenz gegenüber Antidepressiva. Die internationale BRIDGEStudie hat aufgezeigt, dass diese versteckten unterschwelligen bipolar Depressiven ein weltweites Problem bilden [1]. Sie werden voraussichtlich in der künftigen DSM-5 und ICD-11 in ihrer Existenz anerkannt, und es besteht eine gute Aussicht, sie damit viel früher und besser zu identifizieren.

Heute werden viele bipolaren Erkrankungen nicht nur mit einer Verspätung von vielen Jahren, während denen sie als Depressionen gelten, diagnostiziert [7, 8], sondern in ihrer Häufigkeit, die zwischen 40-50\% aller affektiven Störungen liegt, gewaltig unterschätzt.

Die Früherkennung der Bipolarität hat schließlich auch eine große Bedeutung für die Prävention in der Jugend (Schulversagen, Delinquenz, Sucht, Adipositas) und im Alter für die Prävention gegen Demenz, welche gehäuft vorkommt und durch Lithium reduziert werden kann. Fragebogen (MDQ, HCL-32 R1) zur Selbstbeurteilung der Hypomanie können diagnostisch hilfreich und zeitsparend sein.

Vorsicht geboten ist bei einer Diagnose bipolarer Störungen im Kindesalter. Obwohl 85\% der Störungen vor dem 20. Altersjahr beginnen, sind genaue Diagnosen meist erst in der Präpubertät ab etwa dem 11. Altersjahr möglich $[5,6]$.

Unglücklicherweise gibt es auch noch keine therapeutischen Studien über die unterschwelligen bipolaren Erkrankungen. Es ist daher nicht möglich für diese große Gruppe von Patienten evidenz- cher und kann am Ende jeder weiteren Phase in Bipolarität kippen. Dieses Risi- 
basierte therapeutische Empfehlungen zu geben. Es ist z. B. problematisch, kurzschlüssig atypische Neuroleptika zu verordnen. Die Patienten sollten vorläufig weiterhin mit Antidepressiva behandelt werden, auch wenn Therapieresistenz häufiger zu erwarten ist. Präzise Behandlungsstudien bleiben ein wichtiges zukünftiges Forschungsgebiet.

\section{Korrespondenzadresse}

Prof. Dr. Dr. J. Angst

Forschungsabteilung Psychiatrische,

Universitätsklinik Zürich

Zürich, Schweiz

jules.angst@bli.uzh.ch

Interessenkonflikt. Der korrespondierende Autor gibt an, dass kein Interessenkonflikt besteht.

\section{Literatur}

1. Angst J, Cui L, Swendsen J et al (2010) Major depressive disorder with subthreshold bipolarity in the National Comorbidity Survey Replication. Am J Psychiatry 167:1194-1201

2. Bauer M, Glenn T, Rasgon N et al (2011) Decreasing the minimum length criterion for an episode of hypomania: evaluation using self-reported data from patients with bipolar disorder. Eur Arch Psychiatry Clin Neurosci 261:341-347

3. Bauer M, Rasgon N, Grof P et al (2005) Mood changes related to antidepressants: a longitudinal study of patients with bipolar disorder in a naturalistic setting. Psychiatry Res 133:73-80

4. Bauer M, Rasgon N, Grof P et al (2006) Do antidepressants influence mood patterns? A naturalistic study in bipolar disorder. Eur Psychiatry 21:262269

5. Duffy A (2007) Does bipolar disorder exist in children? A selected review. Can J Psychiatry 52:409417

6. Duffy A, Alda M, Crawford L et al (2007) The early manifestations of bipolar disorder: a longitudinal prospective study of the offspring of bipolar parents. Bipolar Disord 9:1-11

7. Hirschfeld RMA, Calabrese JR, Weissman MM et al (2003) Screening for bipolar disorder in the community. J Clin Psychiatry 64:53-59

8. Hirschfeld RMA, Lewis L, Vornik LA (2003) Perceptions and impact of bipolar disorder: how far have we really come? Results of the National Depressive and Manic-Depressive Association 2000 Survey of individuals with bipolar disorder. J Clin Psychiatry 64:161-174

9. Zimmermann P, Brückl T, Nocon A et al (2009) Heterogeneity of DSM-IV major depressive disorder as a consequence of subthreshold bipolarity. Arch Gen Psychiatry 66:1341-1352

Nervenarzt 2012 $\cdot 83: 904-906$

DOI 10.1007/s00115-012-3579-5

Online publiziert: 27. Juni 2012

๑) Springer-Verlag 2012

\section{E. Severus}

Klinik und Poliklinik für Psychiatrie und Psychotherapie, Universitätsklinikum Carl Gustav Carus, TU Dresden

\section{Sind bipolare Erkrankungen viel häufiger als bisher angenommen? Kontra}

In den letzten Jahren sind von verschiedenen im Bereich der bipolaren Störungen tätigen Wissenschaftlern Arbeiten veröffentlicht worden, die nahelegen, dass bipolare Erkrankungen viel häufiger als bisher angenommen vorkommen. In einer dieser Arbeiten wird nunmehr von einer Lebenszeitprävalenz von ca. $9 \%$ ausgegangen (bisher ca. 2-3\%) [1]. Im Gegenzug hierzu würde sich die Prävalenz rein unipolarer Depressionen deutlich reduzieren, mit nur unwesentlich mehr unipolaren Depressionen als bipolaren Erkrankungen [1, 2, 3].

Begründet wird dieses damit, dass die aktuellen Kriterien nach DSM-IV-TR, dem amerikanischen Klassifikationssystem psychiatrischer Erkrankungen, für das Vorliegen einer hypomanen Episode zu eng gefasst seien und zwar, so die BRIDGE-Studie, sowohl was die Mindestdauer, die Kernsymptomatik, die Konsequenzen als auch die Ausschlusskriterien für hypomane Episoden angeht (DSM-IVTR; [2]). So würden, unter den neu vorgeschlagenen, weiter gefassten Kriterien, auch aktuell depressive Patienten, die in der Vergangenheit, z. B. im direkten zeitlichen Zusammenhang mit der Einnahme von Amphetaminen, eine Phase gesteigerter Aktivität entwickelten, bei gleichzeitig vermindertem Schlafbedürfnis, vermehrtem Redebedarf und Größenideen, ohne dass hierbei begleitend eine Veränderung der Stimmung vorliegen müsste, die Diagnosekriterien einer bipolaren Erkrankung erfüllen. Auch würde dies auf Patienten/- innen zutreffen, die z. B. im Rahmen einer emotional-instabilen Persönlichkeitsakzentuierung/-störung wiederholt Zeiten kennen, in denen sie gereizter sind, aufgeregter, gesprächiger und sich dabei gleichzeitig aktiver fühlen. Es ist dann auch folgerichtig, dass in der BRIDGE-Studie, erst unter Hinzufügung dieser neuen Gruppe „bipolarer" Patienten zu den nach DSM-IVTR bipolar erkrankten Patienten, sowohl ein aktueller Substanzmissbrauch als auch eine Borderline-Persönlichkeitsstörung zu vermeintlichen Risikofaktoren für bipolare Erkrankungen werden, in Abgrenzung zu rein unipolaren Depressionen. Es wird nun angeführt, dass diese neue Gruppe „bipolarer" Patienten durchaus auch andere Charakteristika mit bipolaren Erkrankungen nach DSM-IV-TR teilt, in Abgrenzung zu unipolaren Depressionen, wie z. B ein früheres Ersterkrankungsalter, eine größere Anzahl an stattgehabten depressiven Episoden, das häufigere Vorkommen von depressiven Mischzuständen oder auch eine positive Familienanamnese hinsichtlich bipolarer Erkrankungen bei Verwandten 1. Grades. Allerdings ist z. B. von Patienten mit Borderline-Erkrankung bekannt, dass für letztere hinsichtlich der Mehrzahl dieser Variablen Ähnliches gilt [4], sodass diese Variablen damit nur bedingt geeignet erscheinen, als externe Validatoren für diese neue Gruppe „bipolarer“ Patienten zu fungieren.

Vor dem Hintergrund des oben Beschriebenen mag es auch von Interesse sein, dass in der Überarbeitung des 